\title{
Multi-objective evolutionary algorithm for operating parallel reservoir system
}

\author{
Li-Chiu Chang ${ }^{\mathrm{a}}$, Fi-John Chang ${ }^{\mathrm{b}, *}$ \\ ${ }^{a}$ Department of Water Resources and Environmental Engineering, Tamkang University, Taiwan, ROC \\ ${ }^{\mathrm{b}}$ Department of Bioenvironmental Systems Engineering, National Taiwan University, Taiwan, ROC
}

\section{A R T I C L E I N F O}

\section{Article history:}

Received 8 February 2009

Received in revised form 7 June 2009

Accepted 29 July 2009

This manuscript was handled by G. Syme, Editor-in-Chief, with the assistance of

Soroosh Sorooshian, Associate Editor

\section{Keywords:}

Optimization

Simulation model

Reservoir system

Multi-objective evolutionary algorithm

\begin{abstract}
S U M M A R Y
This paper applies a multi-objective evolutionary algorithm, the non-dominated sorting genetic algorithm (NSGA-II), to examine the operations of a multi-reservoir system in Taiwan. The Feitsui and Shihmen reservoirs are the most important water supply reservoirs in Northern Taiwan supplying the domestic and industrial water supply needs for over 7 million residents. A daily operational simulation model is developed to guide the releases of the reservoir system and then to calculate the shortage indices (SI) of both reservoirs over a long-term simulation period. The NSGA-II is used to minimize the SI values through identification of optimal joint operating strategies. Based on a 49 year data set, we demonstrate that better operational strategies would reduce shortage indices for both reservoirs. The results indicate that the NSGA-II provides a promising approach. The pareto-front optimal solutions identified operational compromises for the two reservoirs that would be expected to improve joint operations.
\end{abstract}

(c) 2009 Elsevier B.V. All rights reserved.

\section{Introduction}

Meeting water supply needs to fast growing populations requires new tools that consider domestic, industrial, and agricultural water uses. In areas where water scarcity exists, procedures must be developed to address the existing and future needs of domestic and industrial needs of fast growing cities without shortchanging agriculture users. In Taiwan, the non uniform distribution of precipitation in dry and wet seasons and the need to retain rainfall for human uses has led to reservoir construction. Water storage in reservoirs, when well managed, will meet user needs, but new challenges in sustainable management are emerging as competition of available water supplies increases. In the past, reservoirs were constructed and managed individually. The practical and environmental constraints to new reservoir construction require that individual reservoir operation must transition to multiple reservoirs, water resources systems approaches. Reservoir systems are challenging because the dynamic, non-linear, and stochastic characteristics of reservoirs must now be addressed from a systems view. Reservoir operation modeling has been subject intense research presenting various optimization techniques (Labadie, 2004; Loucks et al., 1981; Tu et al., 2008; Yeh, 1985). Many water resource systems are characterized by multiple objectives that often conflict and compete with one another. Tradeoff methodologies have shown promise as effective means for considering non-

\footnotetext{
* Corresponding author. Tel.: +8862 23639461; fax: +886223635854.

E-mail address: changfj@ntu.edu.tw (F.-J. Chang).
}

commensurate objectives that are subjectively compared in operation determinations (Cohon and Marks, 1975). To handle multiple objectives, many studies of reservoir operation have used either a weighting approach or constraint method to optimization based on dynamic programming (DP), linear programming (LP) and nonlinear programming (NLP), stochastic dynamic programming (Labadie, 2004; Yeh, 1985).

In the multiobjective approach the number of possible solutions are presented as a Pareto-optimal front, instead of a single optimal solution. To deal with the Pareto front, classical optimization methods suggest converting a multi-objective problem to a single-objective problem by emphasizing one particular Pareto-optimal solution at a time. Using this approach requires multiple iterations of simulation models with the hope of identifying better solutions (Deb, 2002). To address the problems of this brute force approach there is an increasing interest in the use of biologicallybased evolutionary algorithms (EAs) for solving problems having interdependence among the decision variables (Cai et al., 2001; Chang, 2008; Chang and Chang, 2001; Chang et al., 2005; Chen et al., 2007; Chen and Chang, 2009; Chiu et al., 2007; Huang and Yuan, 2004; Huang et al., 2002; Jin et al., 2008; Keedwell and Khu, 2006; Kollat and Reed, 2007; Reddy and Kumar, 2006; Yang et al., 2007). Genetic algorithms (GAs) form one of the most promising of these EAs. The 'population-by-population approach' is the most important characteristic of GAs when compared to the 'pointby-point method' of traditional optimization techniques (Goldberg and Kuo, 1987). EA multi-objective optimization algorithms are similar to single objective EAs in that all genetic algorithms search 
complex problem spaces by using a process that is analogous to Darwinian natural selection (Deb, 2002; Srinivas and Deb, 1994). The key feature of these algorithms is the population based approach, which enables identification of a diverse set of Pareto-optimal solutions in a single simulation run. Further, a single run can illustrate trade-off relationships between objectives. These tradeoff solutions have been found to improve traditional optimization and are termed non-dominated or Pareto-optimal solutions. The tradeoff information is very useful in making a sound decision for alternative options.

The non-dominated sorting genetic algorithm (NSGA-II) proposed by Deb et al. $(2002,2000)$ is one of the contemporary multi-objective EAs that contains few tuning parameters and exhibits high performance, making it more attractive for real-world applications. In real-world applications it is often more persuasive to demonstrate the descriptive nature of the system behavior with a set of non-inferior optimal solutions than with a single objective optimal solution. The EA approach has been found to provide a better spread of solutions and to converge better to the nondominated set for the test problems (Kollat and Reed, 2005). This is particularly true for hydrological problems, such as in water distribution networks (Atiquzzaman et al., 2006; Jin et al., 2008), reservoir operation (Kim et al., 2006), parameter optimization (Bekele and Nicklow, 2007; Nazemi et al., 2006), wastewater systems (Fu et al., 2008), and groundwater monitoring (Kollat and Reed, 2007).

The purpose of this paper is to solve a multi-reservoir system optimization by coupling an operational simulation model with an EA. The Feitsui and Shihmen reservoirs, the two most important water supply reservoirs in Northern Taiwan, are the focus of this analysis. The Shihmem reservoir has a serious water shortage problem while the Feitsui reservoir has a surplus supply. They are parallel systems and are operated independently by two government departments unless an emergency (flooding or serious drought) shifts management to the Water Resource Agency. The opportunity exists to address the existing water supply problem through development of a joint operational strategy. The EA selected is the NSGA-II, which is coupled with reservoir operation simulations to optimize joint operations. The shortage index (SI) proposed US Army Corps of Engineers was adopted as the criteria for evaluating the reservoir performance. The Pareto-front results present a range of operational choices that are practical solutions that can find support of local decision makers.

\section{The multi-objective evolutionary algorithm}

A number of multi-objective evolutionary algorithms (MOEAs) have been suggested in the last two decades, and the non-dominated sorting genetic algorithm II (NSGA-II) is one of the promising MOEAs and has been successfully applied in many fields. The initial non-dominated sorting genetic algorithm (NSGA) proposed by Srinivas and Deb (1994) could find multiple Pareto-optimal solutions in one simulation run for multi-objective optimization problems. The NSGA-II, is an improved version of NSGA developed to address issues of computational complexity as well as to provide an explicit mechanism for diversity preservation (Deb et al., 2000).

The NSGA-II algorithm consists of five operators: initialization, fast non-dominated sorting, crossover, mutation and the elitist crowded comparison operator. The major difference between NSGA-II and other EAs is the method of operator selection. The NSGA-II uses the non-dominated sorting and ranking selection with the crowded comparison operator (Deb et al., 2000). Three new innovations are outlined in the following:

(1) Fast non-dominated sorting: The fast non-dominated sorting approach has a better book-keeping strategy to speed up the non-dominated sorting process and reduce the computation complexity.

(2) Crowding distance calculation: NSGA-II adopts a crowding distance to measure the density of individuals in the same front. The overall crowding distance is calculated as the sum of individual distance values corresponding to each objective. Besides the non-domination rank, the crowding distance of each individual is also calculated by the average Euclidean distance between the individual and those adjacent individuals in terms of each of the m objectives.

(3) Crowded comparison operator: This operator guides the selection process at various stages towards a uniformly spread-out Pareto-optimal front. The crowding distance is applied to select one with a greater crowding distance from two individuals in the same front. The elitist crowded comparison operator combines offspring population members with parent population in the selection process that significantly speeds up to capture the previously found nice solutions.

The implementation of the NSGA-II in this study uses the following method:

Step 1: Randomly initialize a population $P_{0}$ of size $N$ and evaluate fitness values; implement the fast non-dominated sorting to divide the population into different ranks; calculate crowding distances of the population.

Step 2: Implement a binary tournament selection, simulated binary crossover (SBX) and polynomial mutation operators to generate an offspring population $Q_{0}$ of size $N$.

Step 3: For every generation $t$, evaluate the fitness values of $Q_{t}$; combine $Q_{t-1}$ and $Q_{t}$ into an intermediate population $P_{t}$ of size $2 \mathrm{~N}$; implement the fast non-dominated sorting to divide this combined population into different ranks; calculate crowding distances of the population.

Step 4: Select a new parent population $P_{t+1}$ of size $N$ from $P_{t}$ using a binary tournament selection; generate an offspring population $Q_{t+1}$ through simulated binary crossover and polynomial mutation operators; evaluate their fitness values.

Step 5: Repeat Steps 3 and 4 until the stop criterion is reached.

Handling constraints present problems in EAs and many solutions have been proposed, including penalty functions, separation of objectives and constraints, repair algorithms, and hybrid methods (Coello, 2002). Optimization of the joint reservoir operating rules involves both variables and constraints in this paper. We adopt a simple constraint-handling strategy suggested by Deb et al. (2002) that is well suited for EAs. The strategy assigns each infeasible solution an overall constraint violation value while a feasible solution has a zero constraint violation. Any feasible solution has a better non-domination rank than all infeasible solutions. Correspondently, an infeasible solution with a smaller overall constraint violation has a better rank.

\section{Two-parallel-reservoirs system formulation}

In this study the NSGA-II is coupled with a reservoir simulation model to search optimal operating strategies these two critical water supplies in northern Taiwan. The Tanshui River is the third longest river in Taiwan with a length of $158.7 \mathrm{~km}$, watershed area of $2726 \mathrm{~km}^{2}$ and annual runoff of 5860 million $\mathrm{m}^{3}$ (MCM). The Tanshui River three major tributaries, the Keelung River, the Hsintien River and the Tahan River. The metropolitan area of Taipei, with a population of 7.5 million, is located in the Tanshui River basin, 


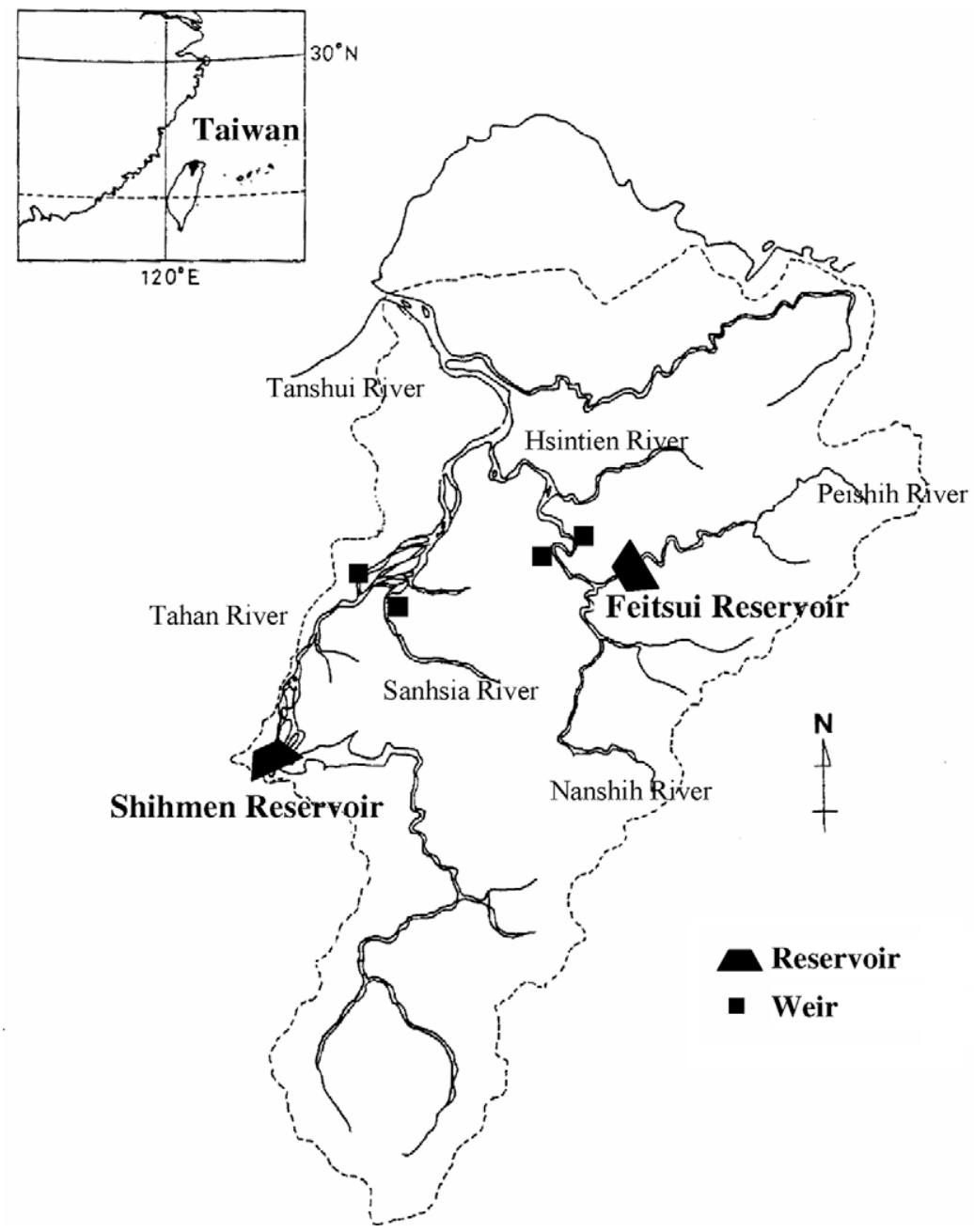

Fig. 1. Locations of Tanshui River Basin and the two-reservoir system.

Table 1

The water supply and demand information of two-reservoir system.

\begin{tabular}{|c|c|c|c|c|}
\hline \multirow[t]{2}{*}{ System } & \multicolumn{2}{|c|}{ Hsintien River } & \multirow{2}{*}{$\begin{array}{l}\text { Tahan River } \\
\text { Shihmen }\end{array}$} & \multirow{2}{*}{$\begin{array}{l}\text { Sanhsia River } \\
\text { Sanhsia weir }\end{array}$} \\
\hline & $\begin{array}{l}\text { Peishih } \\
\text { Feitsui }\end{array}$ & $\begin{array}{l}\text { Nanshih } \\
-\end{array}$ & & \\
\hline Watershed area $\left(\mathrm{km}^{2}\right)$ & 310 & 332 & 763 & 137 \\
\hline Annual runoff (MCM) & 951 & 1119 & 1458 & 292 \\
\hline Effective storage (MCM) & 336 & - & 235 & 0.5 \\
\hline Maximum water level (m) & 172.5 & - & 251 & - \\
\hline Minimum water level (m) & 110 & - & 195 & - \\
\hline Ecological baseflow $\left(\mathrm{m}^{3} / \mathrm{s}\right)$ & 2 & & 2.3 & 1 \\
\hline Irrigation water demand (MCM/yr) & 0 & & 517 & 60 \\
\hline M \& I water demand (MCM/yr) & 1164 & & 497 & 369 \\
\hline
\end{tabular}

Fig. 1. The two reservoirs are located on major tributaries that join to form the Tanshui River. The main features of this basin are summarized in Table 1 and described as follows:

- The Hsintien River has two major tributaries, the Peishih River and Nanshih River, for which the average annual runoff is 950 and $1110 \mathrm{MCM}$, respectively. The Feitsui reservoir is located in the Peishih River with an effective storage capacity of 336 MCM and serves municipal and industrial (M \& I) water use for a population of 3.83 million of metropolitan Taipei.

- The Shihmen reservoir, located upstream area of the Tahan River with an effective storage capacity of $235 \mathrm{MCM}$ and annual runoff of $1410 \mathrm{MCM}$, supplies agricultural and municipal, industrial water use for a population of 1.76 million of Taoyuan county and a part of Taipei county.

- Another water district of Taipei county which includes eleven administrative districts with a population of 1.91 million is also considered. At present, its water supply is provided by Banhsin plant, which uses water from both the Sanhsia River and the Shihmen reservoir. The Sanhsia River has a drainage area of $137 \mathrm{~km}^{2}$ and annual runoff of $260 \mathrm{MCM}$, with an effective storage capacity of a single weir of 0.5 MCM.

The Feitsui and Shihmen reservoirs are operated independently according to operating rules implemented by two agencies, the Taipei city government and the Northern Region Water Resource 
Office, Water resource Agency, in the Ministry of Economic Affairs. In emergencies such as a serious drought or flooding the Water Resource Agency has the authority to operate both reservoirs for the emergency period.

\section{The available data}

The data available for simulation modeling and EA analysis include historical daily inflow data, reservoir properties, rule curves, and expected water demand. Daily inflow data is available for the past 49 years (1958-2006) and were used to simulate the dynamics of the multi-reservoir system and evaluate the model performance. The future water demand was set at the target value for the year of 2021 .

\section{The strategy of joint operation}

The Shihmen reservoir has an existing water supply deficit while the Feitsui reservoir has surplus water. There is a channel that provides water from the Feitsui reservoir to the Banhsin water treatment facility. The Banhsin plant is a part of the Shihmen reservoir water supply district and with connection to the Feitsui reservoir can alleviate Shihmen reservoir shortages. The proposed model uses a common water district management approach as the method to consolidate joint water supplies and meet the increasing water demand by municipal and industrial users. Fig. 2 provides a schematic representation of the two-reservoir system.

Considering the existing management structure and available joint operations approaches this analysis is focused on increasing the efficiency of use of Feitsui reservoir supplies with operations based on a joint operations rule curve that focuses on the Feitsui reservoir. Basically, this operational scheme determines if the Feitsui reservoir should supply Banhsin plant.

The simulation procedure of the joint operation is that the water supply for the common district (i.e. Banhsin plant) will take from the Sanhsia River first and the insufficient amount of water is then provided by the Feitsui reservoir or Shihmen reservoir. The water level of the Feitshui reservoir is the key to determine which reservoir should supply water to the common district. When the water level of the Feitshui reservoir is above the joint operating rule curve, the Feitshui reservoir would supply water; otherwise, the Shihmen reservoir should supply water to the common district. According to this strategy, the following simulation model is proposed.

\section{Simulation model}

Simulation has capability of capturing uncertainty, complex system dynamics and large-scale systems. Simulation models are useful for describing and analyzing the behavior of reservoir release operations through the evaluation of various scenarios. We developed a simulation model for daily operations of the tworeservoir system based on existing reservoir rule curves and the joint operating rule curve derived from NSGA-II. The operational assumptions for water supply distribution for three water districts are explained in detail as follows:

(1) The Banhsin plant will take water from the Sanhsia weir first. The capacity of this weir is only 0.5 MCM, which is much less than the water demand of the common district. To meet needs water must be provided by the Feitsui or Shihmen reservoirs. The relationships between the inflow (ISan) and the water demands $\left(D_{I R}^{S a n}, D_{\mathrm{M} \& a m p ; \mathrm{I}}^{B}\right)$ are presented in the following equations.

$$
\begin{aligned}
& I^{\operatorname{SanU}}(t)=\operatorname{Min}\left[0.5, \operatorname{Max}\left(I^{\operatorname{San}}(t)-D_{I R}^{\operatorname{San}}(t), 0\right)\right] \\
& R^{B}(t)=\operatorname{Min}\left[\mu^{F} D_{\mathrm{M} \& \mathrm{I}}^{B}(t), I^{\operatorname{SanU}}(t)\right] \\
& D_{\mathrm{M} \& \mathrm{I}}^{B R}(t)=\operatorname{Max}\left[0, \mu^{F} D_{\mathrm{M} \& \mathrm{I}}^{B}(t)-R^{B}(t)\right]
\end{aligned}
$$

where $I^{S a n}, I^{S a n U}$ and $D_{I R}^{\text {San }}$ are the inflow, usable inflow and irrigation demand of the Sanhsia River; $D_{\mathrm{M} \& a m p ; \mathrm{I}}^{B}$ is M \& I water demand of the Banhsin Plant; $\mu^{F}$ is the percentage of required water quantity release according to the rule curves of the Feitsui reservoir; $R^{B}$ is the release taken from the Sanhsia weir; $D_{\mathrm{M} \& a m p ; \mathrm{I}}^{B R}$ is the shortage of $\mathrm{M} \& \mathrm{I}$ water demand in Banhsin Plant that should be replenished by the Feitsui or Shihmen reservoirs.

(2) The Feitsui reservoir and Nanshih River serve their water district based on the following policies. The operating policies of the Feitsui reservoir would be (1) to meet demand

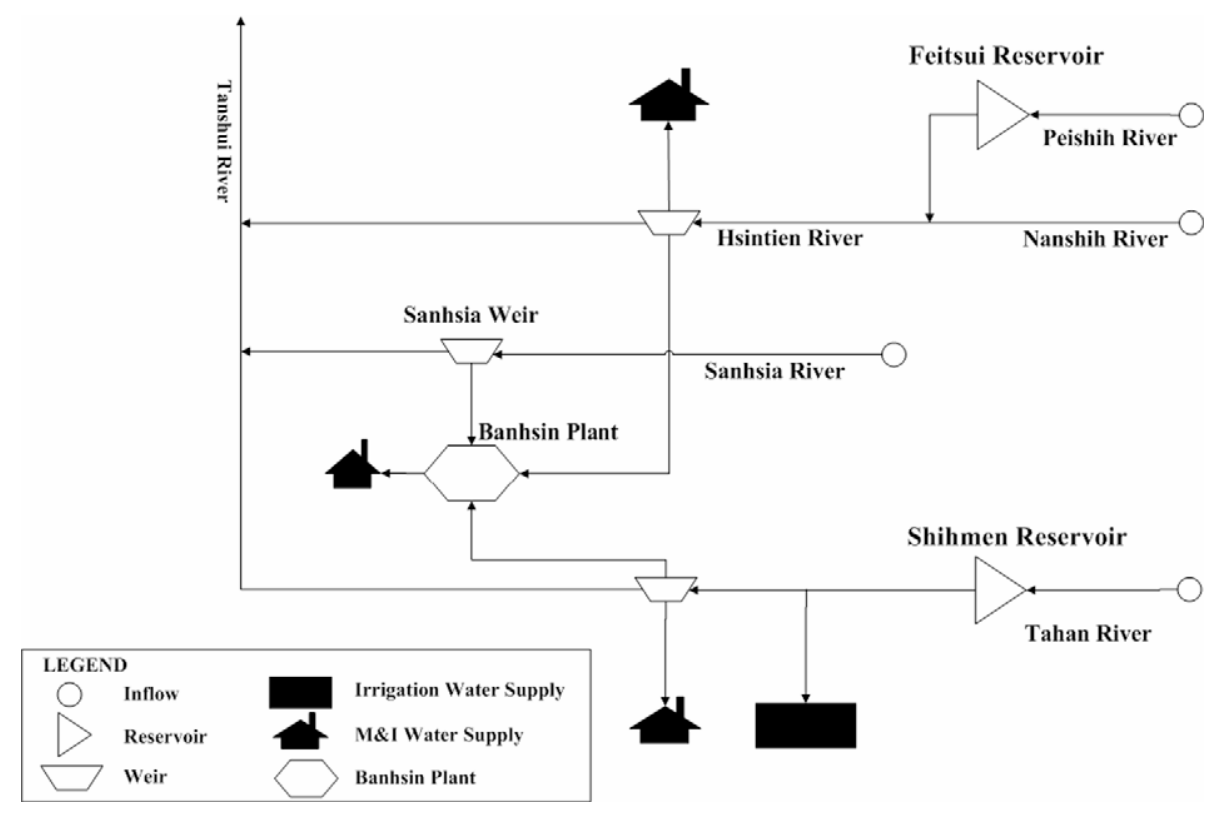

Fig. 2. Schematic diagram of the two-reservoir system. 


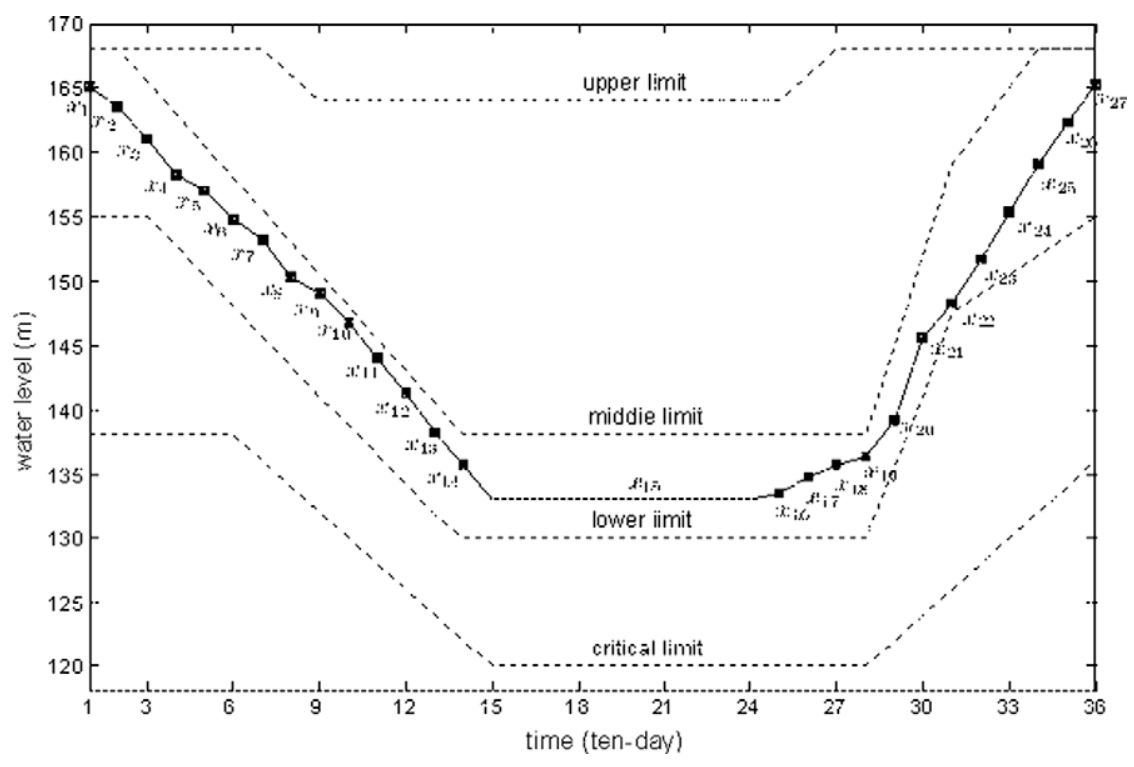

Fig. 3. Decision variables definition of joint operating rule curve.

if the water level is above the lower limit (2) to reduce water supply for demand by $10 \%$ if the water level is between the lower limit and critical limit, and (3) to reduce supply by $30 \%$ if the water level is below the critical limit. The Feitsui reservoir could also serve the common district if its water level is above the joint operating rule (shown as Fig. 3), Consequently, the water release of the Feitsui reservoir is divided into two situations as follows:

(a) The water level is above the joint operating rule curve:

$R^{F}(t)=\mu^{F} D_{\mathrm{M} \& \mathrm{I}}^{F}(t)+D_{\mathrm{M} \& \mathrm{I}}^{B R}(t)$

(b) The water level is below the joint operating rule curve:

$R^{F}(t)=\mu^{F} D_{\mathrm{M} \& \mathrm{I}}^{F}(t)$
$S^{F}(t)=S^{F}(t-1)+I^{F}(t)-E^{F}(t)+\operatorname{Min}\left[I^{N a n}(t)-R^{F}(t)-R_{E c 0}^{F}, 0\right]$

$S_{\min }^{F} \leqslant S^{F}(t) \leqslant S_{\max }^{F}$

where $S^{F}, D_{\mathrm{M} \& a m p ; \mathrm{I}}^{F}, R^{F}, I^{F}$ and $E^{F}$ are the storage, M \& I water demand, release, inflow and evaporation loss of the Feitsui reservoir, respectively; $R_{E c o}^{F}$ is the required ecological base flow taken from Feitsui; $I^{N a n}$ is the inflow of the Nanshih River.

(3) The Shihmen reservoir has a water district for irrigation adding agriculture to municipal and industrial water demands. The Shihmen reservoir also supplies the common water district not served by the Feitsui reservoir. The operating policies of the Shihmen reservoir would be (1) to meet demand if the water level is above its critical limit and (2) to reduce $\mathrm{M} \& \mathrm{I}$ demand by $30 \%$ and irrigation demand by $50 \%$ if the water level is below its critical limit. The relationships among the storage (SS), the inflow (IS) and the water demands $\left(D_{I R}^{S}, D_{\mathrm{M} \& a m p ; \mathrm{I}}^{S}, D_{\mathrm{M} \& a m p ; \mathrm{I}}^{B R}\right)$ in two situations are explained as the following equations.

(a) The water level of Feitsui is above the joint operating rule curve:

$R^{S}(t)=\mu_{I R}^{S} D_{I R}^{S}(t)+\mu_{\mathrm{M} \& \mathrm{I}}^{S} D_{\mathrm{M} \& \mathrm{I}}^{S}$ (b) The water level of Feitsui is below the joint operating rule curve:

$R^{S}(t)=\mu_{I R}^{S} D_{I R}^{S}(t)+\mu_{\mathrm{M} \& \mathrm{I}}^{S} D_{\mathrm{M} \& \mathrm{I}}^{S}(t)+D_{\mathrm{M} \& \mathrm{I}}^{B R}(t)$
$S^{S}(t)=S^{S}(t-1)+I^{S}(t)-E^{S}(t)-R^{S}(t)-R_{E c o}^{S}$
$S_{\min }^{S} \leqslant S^{S}(t) \leqslant S_{\max }^{S}$

where $S^{S}, D_{I R}^{S}, D_{\mathrm{M} \& a m p ; \mathrm{I}}^{S}, R^{S}, I^{S}$ and $E^{S}$ are the storage, irrigation water demand, $M \&$ I water demand, release, inflow and evaporation loss of the Shihmen reservoir, respectively; $R_{E c o}^{S}$ is the required ecological baseflow taken from Shihmen; $\mu_{I R}^{S}$ and $\mu_{\mathrm{M} \& a m p ; \mathrm{I}}^{S}$ are the percentage of required irrigation and $\mathrm{M}$ \& I quantity release according to whether the water level is below or above of the critical limit rule curve of the Shihmen reservoir, respectively.

\section{Optimal system formulation}

The purpose of the joint operation is to alleviate the regional water shortage and allow the decision maker to choose the best compromise solutions through consideration of the trade-offs between two reservoirs. Based on our simulation experience, the lower the joint operating rule curve designed for the Feitsui reservoir would create increased water shortage for Feitsui users and a somewhat reduced water shortage for Shihmen users. To consider these effects on water shortage a shortage index (SI) was adopted as the criterion for evaluating the reservoir performance; hence, the objective is to minimize the shortage indices produced by joint reservoir operation.

Minimize $\quad Z_{1}=S I^{F}=\frac{100}{n} \sum_{i=1}^{n}\left(\frac{S I_{i}^{F}}{D_{M \& l}^{F}}\right)^{2}$

Minimize $\quad Z_{2}=S I^{S}=\frac{100}{n} \sum_{i=1}^{n}\left(\frac{S I_{i}^{S}}{D_{M \& 1}^{S}+D_{I R}^{S}}\right)^{2}$

where $S I_{i}$ is annual shortage in $i$ th year (annual demand-annual supply); D is annual demand; $n$ is number of simulating years (i.e. 49 years). Eqs. (12) and (13) are the shortage indices (SI) of Feitsui and Shihmen, respectively.

We use the 10-day averaging period adopted by the Water Resources Agency to simulate reservoir operations and provide data 
for SI calculation (Eqs. (12) and (13)). The joint operating rule curve has 36 variables. To be consistent with the seasonal considerations in rule curve development, we use one variable $\left(x_{15}\right)$ to represent 10 water levels for the wet season and 26 variables $\left(x_{i}\right.$ for $i=1, \ldots, 14,16, \ldots, 27)$ to represent all water levels for the other periods. The decision variables can be reduced to 27 variables considering proportional time spans based on Fig. 3 and the following constraints are:

$x_{i+1} \leqslant x_{i}$ for $i=1, \ldots, 14$

$x_{i-1} \leqslant x_{i}$ for $i=16, \ldots, 27$

$\left|x_{i+1}-x_{i}\right| \leqslant 7$ for $i=1, \ldots, 26$

$\left|x_{1}-x_{27}\right| \leqslant 2$

$x_{i}^{L} \leqslant x_{i} \leqslant x_{i}^{U}$ for $i=1, \ldots, 27$

$x_{15}$ represents the operating rule curve's water level during the wet season, and the water level should be proportional with the time span from the wet season (Eqs. (14) and (15)). Eqs. (16) and (17) confine the difference between consecutive variables to get the reasonable solutions, because the large different means that the Feitsui reservoir could supply much more or less water in the next period and result in unreasonably enlarging variation of water supply. $x^{L}$ and $x^{U}$, respectively, are the critical limit and upper limit rule curves to confine the search space in Eq. (18). The NSGA-II is applied to search the optimal vector function (27 variables) and produce Pareto-optimal solutions for both reservoirs (minimal SI values). The Pareto front is a set of Pareto-optimal (non-dominated) solutions, being considered optimal, if no objective can be improved without sacrificing at least one other objective.

\section{Performance measures}

Multi-objective optimization problems, unlike single-objective problems, have two goals: (1) convergence to the Pareto-optimal front and (2) maintenance of diversity in the Pareto-optimal solutions (Deb et al., 2000). In this case, the actual Pareto-optimal solutions are unknown, so the first performance cannot be measured. The maintenance of diversity can be measures as a set of the optimal solutions that provides a diversity of alternatives, where the diversity metric defined as Eq. (19) (Deb et al., 2000).

$\Delta=\frac{d_{f}+d_{l}+\sum_{i=1}^{N-1}\left|d_{i}-\bar{d}\right|}{d_{f}+d_{l}+(N-1) \bar{d}}$

where $d_{i}$ is the Euclidean distance between consecutive solutions in the Pareto-optimal set of solutions; $\bar{d}$ is the average distance of all distances $d_{i} ; d_{f}$ and $d_{l}$ are distances between the extreme solutions and the boundary solutions ( $d_{f}=d_{l}=0$ in this case).

\section{Results}

We develop a water supply simulation model for the above two-parallel-reservoir system in accordance with Eqs. (1)-(11) and use the NSGA-II to search the optimal Pareto-front solutions for two objective functions (Eqs. (12) and (13)) with 53 constraints (Eqs. (14)-(17)) by using a 49 year historical flow record. Table 2 presents the setting parameters of NSGA-II. As shown, the population size was set to 1000 , and 500 generations where final optimal solutions were quickly obtained and nicely distributed along the Pareto front. Ten search results of the Pareto-front SI values for Feitsui and Shihmen reservoirs and the corresponding SI values for Banhsin plant are shown in Table 3. It is easy to tell that (1) the Feitsui reservoir has much smaller values of SI than those of Shihmen reservoir, which confirms that the Shihmen reservoir has a serious water shortage problem while the Feitsui reservoir has much surplus water; (2) the higher SI value of Feitsui reservoir
Table 2

Parameters of NSGA-II

\begin{tabular}{ll}
\hline NSGA-II parameters & Setting value \\
\hline Number of decision variables & 27 \\
Number of constraints & 53 \\
Number of objective function & 2 \\
Population size & 1000 \\
Generation & 500 \\
Crossover rate & 0.8 \\
Mutation rate & 0.05 \\
Distribution index for SBX & 20 \\
Distribution index for polynomial mutation & 100 \\
\hline
\end{tabular}

will couple with the lower SI values of Shihmen reservoir and Banhsin plant.

Fig. 3 presents a case of the solutions of 27 decision variables (water levels) in the joint operating rule curve of Feitsui reservoir. The Feitsui reservoir would supply water to the common district (the Banhsin plant) only if its water level is above the joint operating rule. The first water level $x_{1}$ determines the highest water level of the joint operating curve, while the fifteenth water level $x_{15}$ decides the lowest water level of the operating curve. Fig. 4 depicts the 1000 Pareto-front SI values of Feitsui and Shihmen reservoirs. It appears the two objectives (SI values) are nicely distributed along the Pareto front and the diversity metric $\Delta$ is small

Table 3

Ten selected SI values of three water districts using NSGA-II and modified 1 rule curves.

\begin{tabular}{|c|c|c|c|c|c|c|}
\hline \multirow[t]{3}{*}{ Case } & \multirow[t]{3}{*}{ Feitsui } & \multicolumn{5}{|l|}{ Method } \\
\hline & & \multicolumn{2}{|l|}{ NSGA-II } & \multicolumn{3}{|c|}{ Rule curves } \\
\hline & & Shihmen & Banhsin & Shihmen & Banhsin & Annotation \\
\hline 1 & 0.043 & 1.768 & 0.809 & 1.831 & 0.836 & Upper \\
\hline 2 & 0.049 & 1.427 & 0.702 & 1.441 & 0.701 & \\
\hline 3 & 0.071 & 1.180 & 0.641 & 1.222 & 0.632 & Middle \\
\hline 4 & 0.102 & 0.964 & 0.549 & 1.047 & 0.596 & \\
\hline 5 & 0.115 & 0.914 & 0.533 & 0.990 & 0.593 & \\
\hline 6 & 0.129 & 0.873 & 0.539 & 0.930 & 0.580 & Lower \\
\hline 7 & 0.155 & 0.770 & 0.512 & 0.849 & 0.566 & \\
\hline 8 & 0.210 & 0.630 & 0.474 & 0.674 & 0.495 & \\
\hline 9 & 0.251 & 0.562 & 0.461 & 0.587 & 0.470 & \\
\hline 10 & 0.286 & 0.527 & 0.459 & 0.529 & 0.463 & Critical limit \\
\hline
\end{tabular}

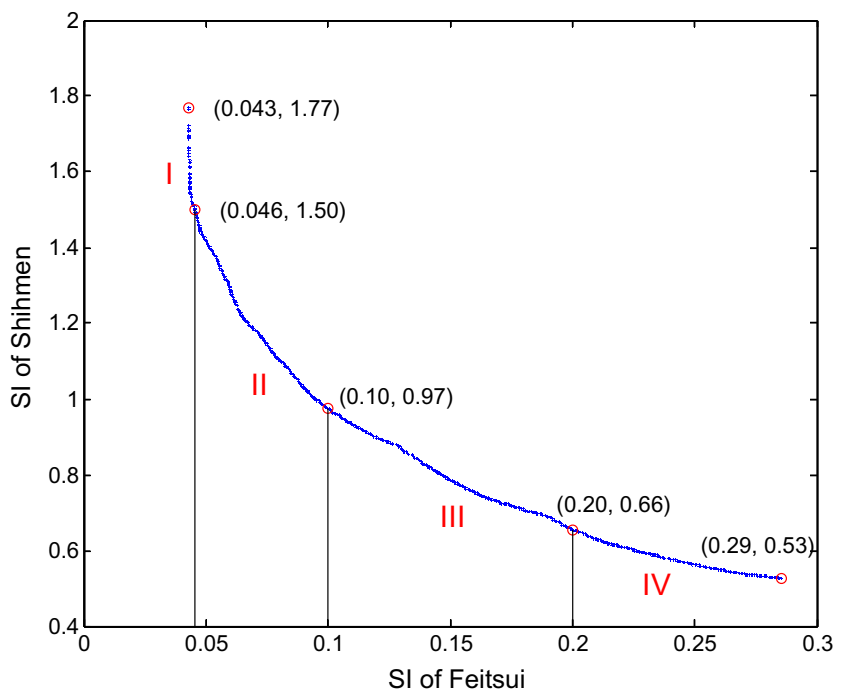

Fig. 4. The Pareto-front SI values of Feitsui and Shihmen reservoirs $(\Delta=0.450)$ 
( $\Delta=0.634$ for ignoring duplicate points and $\Delta=0.450$ for ignoring the distances $d_{i}<0.001$ ), which means the NSGA-II effectively reaches the optimal and diversified solutions. Moreover, we found that the SI values of Shihmen were markedly reduced from 1.77 to about 1.0 (region I and II), while Feitsui only slightly changed from 0.043 to about 0.1 . That means there is great potential for the Feitsui reservoir to provide much more water for the Banhsin plant, which would only slightly increase the water shortage of the Feitsui water supply district but significantly decrease the water shortage of Shihmen water supply district.

A detailed analysis of the results obtained by NSGA-II did identify interesting phenomena that might be useful in the decision making process. Fig. 5 shows the 1000 Pareto-front solutions for $x_{1}$ and $x_{15}$ versus their corresponding SI values. It appears that the water level $x_{1}$ of the Feitsui reservoir only occurs in three ranges $(167 \pm 0.5,156 \pm 1,139.5 \pm 1.5)$, while the water level $x_{15}$ has a wide range $(165-120 \mathrm{~m})$. Higher value of $x_{1}(167 \pm 0.5$, A-B) means that Feitsui retains more water for its own use and does not support water to the common district and low SI values would be expected (0.043-0.13). A lower value of $x_{1}(139.5 \pm 1.5)$ means that Feitsui is willing to provide water to the common district and takes the responsibility of high SI values (greater than 0.2 ). There are five pairs (i.e. A-E cases) of $x_{1}$ and $x_{15}$. In case A, the smallest SI value would be obtained. Cases $B$ and $C$ have the same SI value, but their values of $x_{1}$ and $x_{15}$ are quite different; the same phenomenon is also shown for cases D and E, only a much lower SI value would be given. These results indicate decision makers could have flexible choices in some circumstances to select a conservative (higher $x_{1}$ ) or progressive (lower $x_{1}$ ) operating rule and it would not significantly change the SI value of the Feitsui reservoir (e.g. B\&C and D\&E).

Each SI value of the Pareto-front solutions presents a particular operating rule curve. To illustrate some of these results, the operating rule curves of above five cases are shown in Fig. 6 . The rule curve of case $A$ is relatively close to the upper limit and has a very small SI value $(S I=0.043$ ) for the Feitsui reservoir; the rule

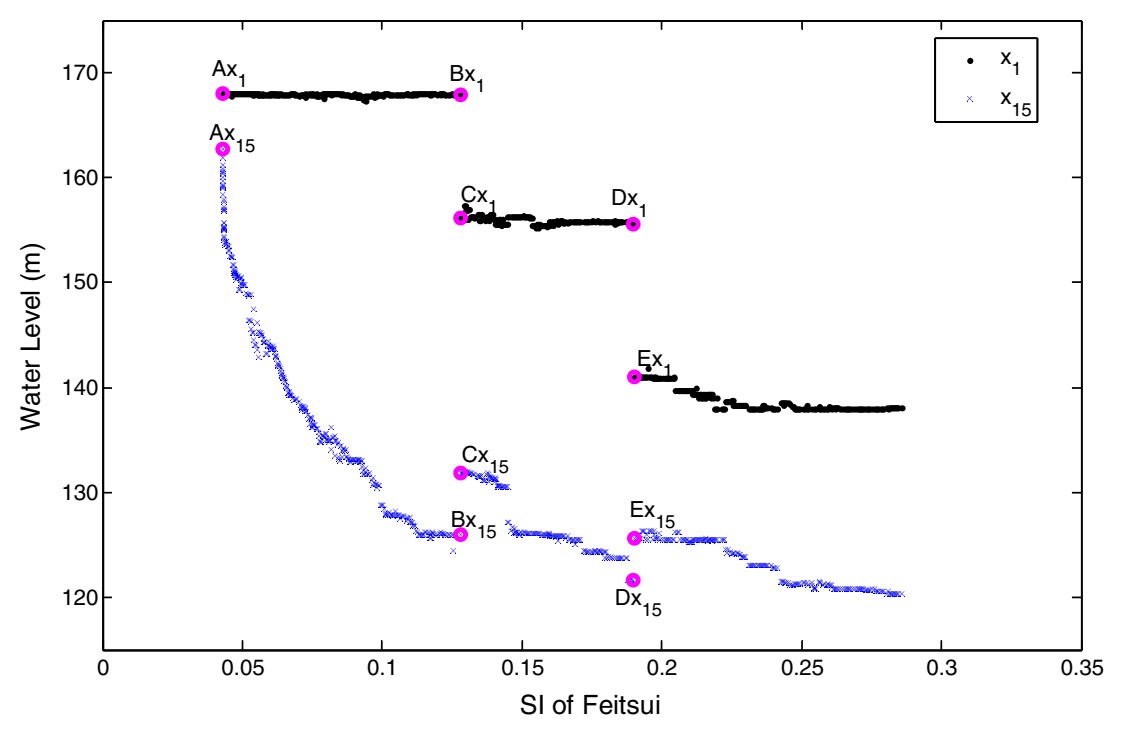

Fig. 5. The highest $\left(x_{1}\right)$ and lowest $\left(x_{15}\right)$ water levels of the Pareto-front solutions for different SI values.

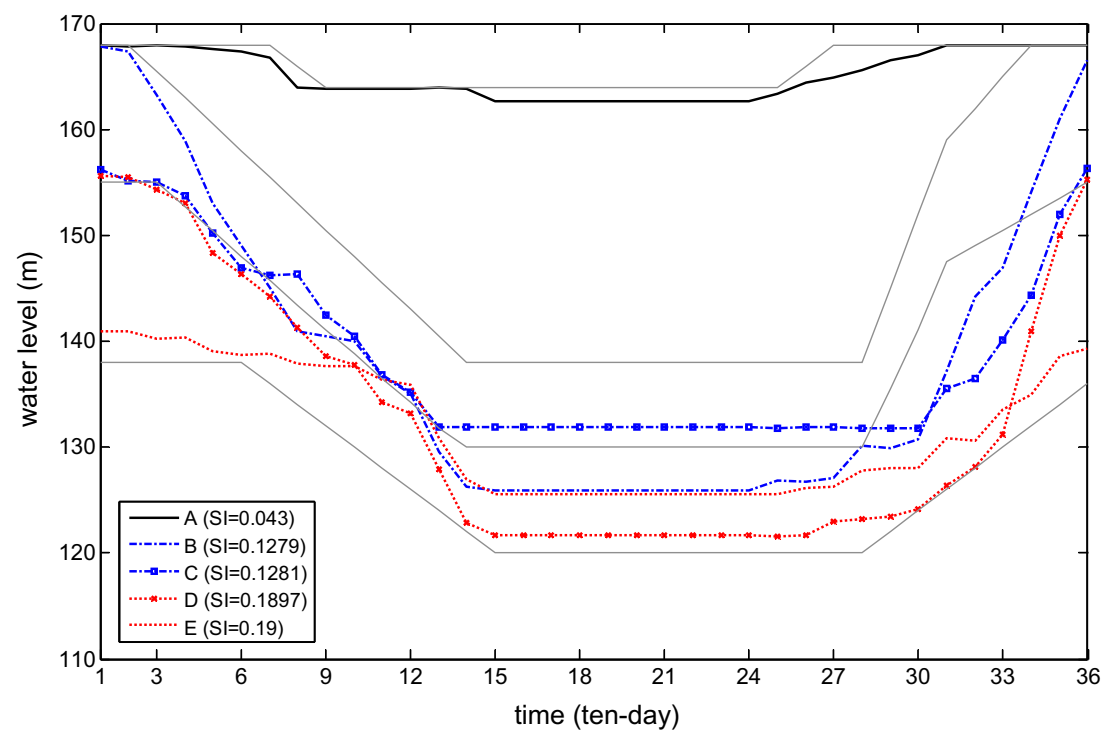

Fig. 6. The operating rule curves with the Feitsui's SI values. 
curves of cases B and C are near the lower limit; while in cases D and $\mathrm{E}$, their rule curves are falling into the range of lower and critical limits and have much higher SI value $(\mathrm{SI}=0.19)$.

For the purpose of comparison, we have simulated the reservoirs' release and calculated the SI values for both reservoirs and Banhsin plant by using a number of operating rule curves, called modified operating rule curves, which are inside the original operating curves (i.e. upper, middle, lower, and critical limits) and have similar shape as those curves to guide the daily operations of this two-reservoir system. The modified rule curves are derived from the original rule curves of Feitsui reservoir and shown as Fig. 7. The comparison results are shown in Table 3 and Fig. 8. The 10 results shown in Table 3 are selected from many simulated results which have similar Feitsui reservoir's SI values as those results of NSGA-II (shown in Table 3). It is easy to tell that the SI values of Shihmen reservoir obtained by NSGA-II are all smaller than those of 10 selected results. Similar results have been obtained for the
Banhsin plant as well. These results indicate the NSGA-II method does obtain better operating rule curves than the original operating curves and would effectively reduce the shortage indexes (SI) of Feitsui and/or Shihmen reservoirs. For instance, when the SI value of Feitsui reservoir equals to 0.155 (case 7$)$, about $10 \%((0.849$ $0.770) / 0.770=0.103 ;(0.566-0.512) / 0.512=0.105)$ improvement of reducing the SI values of the Shihmen reservoir and Banhsin plant is obtained by the NSGA-II as compared with the results obtained by simulating the modified original operating rule curves (Table 3). We like to note that a $10 \%$ improvement of reducing the SI value is a great achievement because this is a lumped water shortage index of 49 years and the large number of water shortages during the drought years is significantly reduced. Fig. 8 clearly demonstrates that as the SI value of Feitsui reservoir is given, the NSGA-II would always have better (smaller) SI values of Shihmen reservoir than those of simulating modified original operating rule curves.

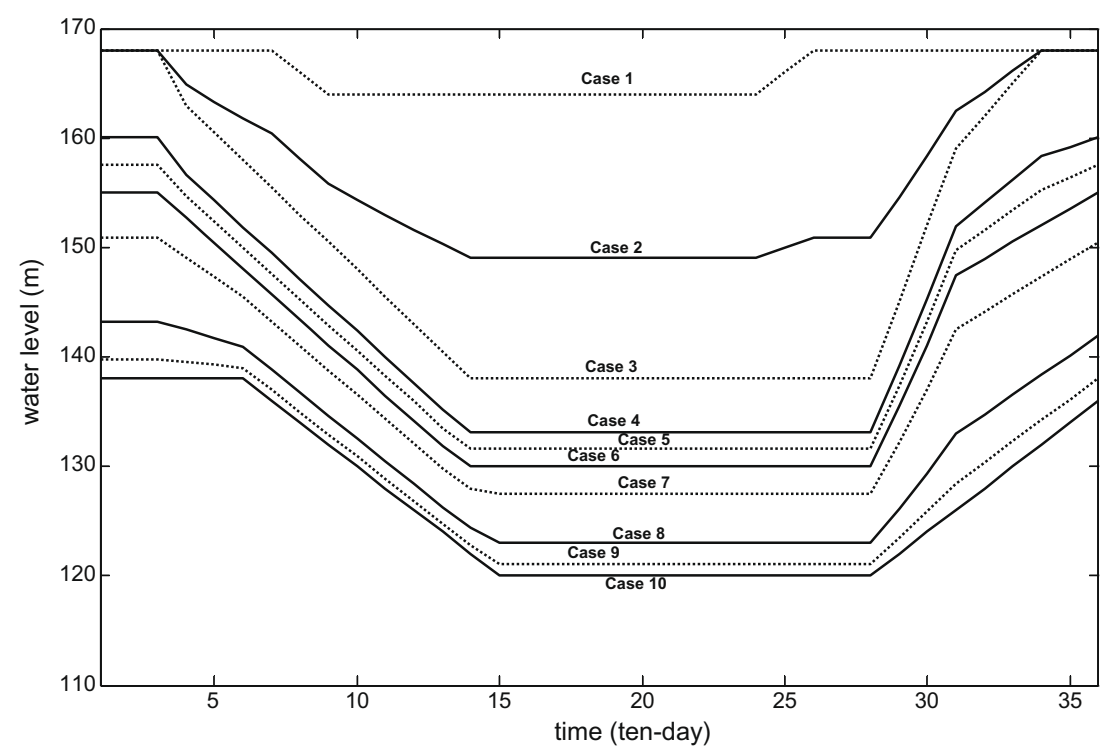

Fig. 7. The 10 selected modified original operating rules.

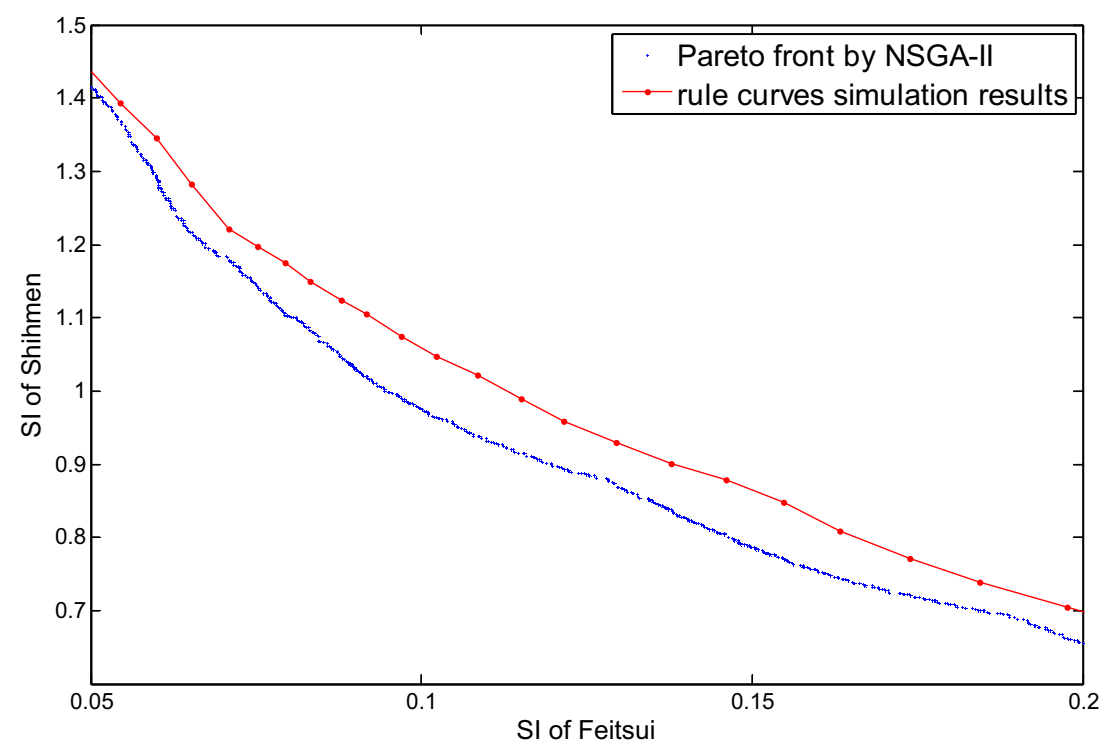

Fig. 8. Comparing SI values of the NSGA-II Pareto front and simulation results. 


\section{Conclusions}

Evolutionary algorithms for multi-objective optimization are gaining significant attention due to the ability of these analysis approaches to address multi-objective problems and discover optimal solutions. The NSGA-II is one of the contemporary multiobjective evolutionary algorithms that exhibit high performance in water resources systems analysis. We developed an integrated simulation model of two parallel reservoirs system, the Feitsui and Shihmen reservoirs, coupled with the NSGA-II to assess optimal joint operating strategies for these reservoirs. The proposed model uses a common water district approach to consolidate reservoir management with the objective of increasing the efficiency of use of water resources from both reservoirs. A daily operational simulation model was applied and shortage indices calculated for a 49 year period. The NSGA-II was used to minimize SI values by identifying joint operating strategies. We demonstrate that there is great potential to alleviate the water shortage problem of this parallel reservoir system through optimizing their operation strategies using this approach. The evolutionary algorithm analysis provides the decision maker with wide range practical alternatives for system management.

The Pareto-front SI values indicate the two-reservoir system can provide practical management solutions. When existing and alternative operational schemes are compared the NSGA-II alternatives produce improved SI values for the Shihmen reservoir. Using alternative management strategies, water shortages during the drought years could be reduced. Our analysis also identified greater decision flexibility that consider the capacity of the Feitsui reservoir to provide additional supplies to Banhsin plant. It is shown that shortages in the Shihmen reservoir would be dramatically decreased with little influence on the capacity of the Feitsui reservoir to meet its water supply needs. We conclude that the NSGA-II is an effective and robust multi-objective technique to identify joint operation strategies that will address critical future sustainability needs in the future.

\section{Acknowledgments}

The author gratefully acknowledges the financial support for this research by the Water Resources Planning Institute, Water Resource Agency, Taiwan, ROC.

In addition, the authors are indebted to Professor Edwin Herricks in UIUC and the reviewers for their valuable comments and suggestions.

\section{References}

Atiquzzaman, M., Liong, S.Y., Asce, M., Yu, X., 2006. Alternative decision making in water distribution network with NSGA-II. Journal of Water Resources Planning and Management 132, 122.

Bekele, E.G., Nicklow, J.W., 2007. Multi-objective automatic calibration of SWAT using NSGA-II. Journal of Hydrology 341 (3-4), 165-176.

Cai, X., Mckinney, D., Lasdon, L.S., 2001. Solving nonlinear water management model using a combined genetic algorithm and linear programming approach. Advanced Water Resources 2 (6), 667-676.

Chang, L.C., 2008. Guiding rational reservoir flood operation using penalty-type genetic algorithm. Journal of Hydrology 354, 65-74.
Chang, L.C., Chang, F.J., 2001. Intelligent control for modelling of real-time reservoir operation. Hydrological Processes 15 (9), 1621-1634.

Chang, Y.T., Chang, L.C., Chang, F.J., 2005. Intelligent control for modeling of realtime reservoir operation, part II: artificial neural network with operating rule curves. Hydrological Processes 19, 1431-1444.

Chen, Y.H., Chang, F.J., 2009. Evolutionary artificial neural networks for hydrological systems forecasting. Journal of Hydrology 367, 125-137.

Chen, L., McPhee, J., Yeh, W.W.G., 2007. A diversified multiobjective GA for optimizing reservoir rule curves. Advances in Water Resources 30 (5), 1082-1093.

Chiu, Y.C., Chang, L.C., Chang, F.J., 2007. Using a hybrid genetic algorithm-simulated annealing algorithm for fuzzy programming of reservoir operation. Hydrological Processes 21, 3162-3172.

Coello, C.C.A., 2002. Theoretical and numerical constraint-handling techniques used with evolutionary algorithms: a survey of the state of the art. Computer Methods in Applied Mechanics and Engineering 191 (11-12), 1245-1287.

Cohon, J.L., Marks, D.H., 1975. A review and evaluation of multiobjective programming techniques. Water Resources Research 11 (2), 208-220.

Deb, K., 2002. Multi-Objective Optimization Using Evolutionary Algorithms. John Wiley and Sons Ltd.

Deb, K., Pratap, A., Agarwal, S., Meyarivan, T., 2002. A fast and elitist multiobjective genetic algorithm: NSGA-II. IEEE Transactions on Evolutionary Computation 6 (2), 182-197.

Deb, K., Pratap, A., Agrawal, S., Meyarivan, T., 2000. A fast elitist non-dominated sorting genetic algorithm for multi-objective: NSGA-II. In: Proceedings of the Parallel Problem Solving from Nature VI Conference, pp. 846-858.

Fu, G., Butler, D., Khu, S.T., 2008. Multiple objective optimal control of integrated urban wastewater systems. Environmental Modelling and Software 23 (2), 225-234.

Goldberg, D.E., Kuo, C.H., 1987. Genetic algorithms in pipeline optimization. Journal of Computing in Civil Engineering 1 (2), 128-141.

Huang, W.C., Yuan, L.C., 2004. A drought early warning system on real-time multireservoir operations. Water Resources Research 40 (6), W06401, doi:10.1029/2003WR002910.

Huang, W.C., Yuan, L.C., Lee, C.M., 2002. Linking genetic algorithms with stochastic dynamic programming to the long-term operation of a multireservoir system. Water Resources Research 38 (2), 401-409.

Jin, X., Zhang, J., Gao, J., Wu, W., 2008. Multi-objective optimization of water supply network rehabilitation with non-dominated sorting genetic algorithm-II Journal of Zhejiang University - Science A 9 (3), 391-400.

Keedwell, E., Khu, S.T., 2006. A novel evolutionary meta-heuristic for the multiobjective optimization of real-world water distribution networks. Engineering Optimization 38 (3), 319-333.

Kim, T., Heo, J.H., Jeong, C.S., 2006. Multireservoir system optimization in the Han River basin using multi-objective genetic algorithms. Hydrological Processes 20 (9), 2057-2075.

Kollat, J.B., Reed, P.M., 2005. The Value of Online Adaptive Search: A Performance Comparison of NSGAII, $\varepsilon$-NSGAII and $\varepsilon$-MOEA. Lecture Notes in Computer Science. Springer Verlag, Heidelberg, D-69121, Germany, Guanajuato, Mexico. pp. 386-398.

Kollat, J.B., Reed, P.M., 2007. A computational scaling analysis of multiobjective evolutionary algorithms in long-term groundwater monitoring applications. Advances in Water Resources 30 (3), 408-419.

Labadie, J.W., 2004. Optimal operation of multireservoir systems: state-of-the artreview. Journal of Water Resources Planning and Management 130 (2), 93-111.

Loucks, D.P., Stedinger, J.R., Haith, D.A., 1981. Water Resources Systems Planning and Analysis. Prentice-Hall, Inc., Englewood Cliffs, NJ.

Nazemi, A., Yao, X., Chan, A.H., 2006. Extracting a Set of Robust Pareto-Optimal Parameters for Hydrologic Models using NSGA-II and SCEM. Evolutionary Computation, 2006. CEC 2006. IEEE Congress on: 1901-1908.

Reddy, M.J., Kumar, D.N., 2006. Optimal reservoir operation using multi-objective evolutionary algorithm. Water Resources Management 20 (6), 861-878.

Srinivas, N., Deb, K., 1994. Muiltiobjective optimization using nondominated sorting in genetic algorithms. Evolutionary Computation 2 (3), 221-248.

Tu, M.Y., Hsu, N.S., Tsai, F., Yeh, W.W.-G., 2008. Optimization of hedging rules for reservoir operations. Journal of Water Resources Planning and Management ASCE 131 (1), 3-13.

Yang, C.C., Chang, L.C., Yeh, C.H., Chen, C.S., 2007. Multiobjective planning of surface water resources by multiobjective genetic algorithm with constrained differential dynamic programming. Journal of Water Resources Planning and Management - ASCE 133 (6), 499-508.

Yeh, W.W.G., 1985. Reservoir management and operation models: a state-of-the-art review. Water Resources Research 21 (2), 1797-1818. 